\title{
A vector spherical harmonic spectral code for linearised magnetohydrodynamics$$
\begin{array}{ll}
\text { D. J. Ivers* } & \text { C. G. Phillips }
\end{array}
$$

(Received 11 July 2001)

\begin{abstract}
Linearised rotating magnetohydrodynamic stability code for the steady axisymmetric basic states of an electrically conducting fluid sphere is described. The code generates compact hybrid angular spectral forms of the magnetic induction, heat and Boussinesq Navier-Stokes equations, using toroidal and poloidal representations of the perturbation vector fields, and vector or scalar spherical harmonic expansions of all fields. The momentum equation may include inertial, Coriolis, buoyancy, viscous and magnetic Lorentz forces. Three subroutines evaluate the spectral interactions of products. There are only six radial functions, which
\end{abstract}

*School of Mathematics \& Statistics, University of Sydney, Australia. mailto:D. Ivers@maths. usyd.edu. au

$\dagger$ Mathematics Learning Centre, University of Sydney

${ }^{0}$ See http://anziamj. austms.org.au/V44/CTAC2001/Ive2 for this article, (C) Austral. Mathematical Soc. 2003. Published 1 April 2003. ISSN 1446-8735 
are discretised using uniform second-order finite differences. The resulting large scale complex non-hermitian generalised eigen- and critical-value problems are solved using inverse and Newton-Raphson iteration methods, respectively. Test results are presented for several models.

\section{Contents}

1 Introduction

C424

2 Hybrid vector spherical harmonic method

$\mathrm{C} 427$

2.1 Toroidal-poloidal fields and spherical harmonics .

$\mathrm{C} 427$

2.2 Vector spherical harmonics . . . . . . . . . . C428

2.3 The hybrid linearised spectral equations . . . . . C430

3 Code structure and radial discretisation

C432

4 Models and results

C435

4.1 Methods for eigen- and critical-value problems . .

$\mathrm{C} 435$

4.2 Rotating thermal convection . . . . . . . . . C436

4.3 Kinematic dynamos with axisymmetric flows . . . C437

4.4 Rotating magnetoconvection . . . . . . . . . . C440

5 Concluding remarks

C441

References

C442

\section{Introduction}

The linearised magnetohydrodynamic stability of an electricallyconducting fluid sphere is considered. Such studies complement 
non-linear dynamically-consistent dynamo calculations. The nonlinear equations for an electrically-conducting Boussinesq fluid in a frame rotating with uniform angular velocity $\boldsymbol{\Omega}$ governing the velocity $\mathbf{v}$, the pressure $p$, the magnetic induction $\mathbf{B}$ and the temperature $\Theta$ are

$$
\begin{gathered}
\rho\left(\partial_{t} \mathbf{v}+\boldsymbol{\omega} \times \mathbf{v}+2 \boldsymbol{\Omega} \times \mathbf{v}\right)=-\nabla P+\mathbf{J} \times \mathbf{B}-\alpha_{\Theta} \Theta \mathbf{g}+\rho \nu \nabla^{2} \mathbf{v} \\
\partial_{t} \mathbf{B}=\eta \nabla^{2} \mathbf{B}+\nabla \times(\mathbf{v} \times \mathbf{B}) \\
\partial_{t} \Theta+\mathbf{v} \cdot \nabla \Theta=\kappa \nabla^{2} \Theta+Q / \rho c_{p} \\
\nabla \cdot \mathbf{v}=0, \quad \nabla \cdot \mathbf{B}=0
\end{gathered}
$$

where $P=p+\frac{1}{2} \rho \mathbf{v}^{2}$ is the modified pressure, $\boldsymbol{\omega}=\nabla \times \mathbf{v}$ is the vorticity, $\mathbf{J}=\nabla \times \mathbf{B} / \mu_{0}$ is the electrical current, $\nu, \eta$ and $\kappa$ are the viscous, magnetic and thermal diffusivities, $\alpha_{\Theta}$ is the thermal expansivity and $Q$ is the rate of heat production per unit volume and $c_{p}$ is the specific heat at constant pressure. The gravitational field may be asymmetric. The diffusivities and specific heat are constants.

The rotating MHD equations (1)-(4), linearised about the steady axisymmetric basic state $\left(\mathbf{v}_{0}, \mathbf{B}_{0}, \Theta_{0}\right)$, with $\mathbf{v}=\mathbf{v}_{0}+\mathbf{v}^{\prime}$, etc, are

$$
\begin{gathered}
\rho\left(\partial_{t} \mathbf{v}^{\prime}+2 \boldsymbol{\Omega} \times \mathbf{v}^{\prime}\right)=-\nabla P^{\prime}+\mathbf{J}_{0} \times \mathbf{B}^{\prime}+ \\
+\mathbf{J}^{\prime} \times \mathbf{B}_{0}-\rho \alpha_{\Theta} \Theta^{\prime} \mathbf{g}+\rho \nu \nabla^{2} \mathbf{v}^{\prime}, \\
\partial_{t} \mathbf{B}^{\prime}=\eta \nabla^{2} \mathbf{B}^{\prime}+\nabla \times\left(\mathbf{v}_{0} \times \mathbf{B}^{\prime}\right)+\nabla \times\left(\mathbf{v}^{\prime} \times \mathbf{B}_{0}\right), \\
\partial_{t} \Theta^{\prime}+\mathbf{v}_{0} \cdot \nabla \Theta^{\prime}+\mathbf{v}^{\prime} \cdot \nabla \Theta_{0}=\kappa \nabla^{2} \Theta^{\prime}+Q^{\prime} / \rho c_{p}, \\
\nabla \cdot \mathbf{v}^{\prime}=0, \quad \nabla \cdot \mathbf{B}^{\prime}=0 .
\end{gathered}
$$

The interaction terms, which arise from the non-linear advection, $\boldsymbol{\omega}_{0} \times \mathbf{v}^{\prime}$ and $\boldsymbol{\omega}^{\prime} \times \mathbf{v}_{0}$, are not required for the test models considered in $\S 4$ and have been omitted. They may be treated similarly to the Lorentz terms. The equations must be supplemented by boundary conditions. Special cases include: thermal convection with $\mathbf{v}_{0}=\mathbf{0}$, 
$\Theta_{0}$ the conduction temperature and $\mathbf{B}_{0}$ omitted; kinematic dynamos with $\mathbf{v}_{0}$ given, $\mathbf{B}_{0}=\mathbf{0}$ and $\Theta_{0}$ omitted; and magnetoconvection with $\mathbf{v}_{0}=\mathbf{0}, \mathbf{B}_{0}$ and $\Theta_{0}(r)$ given. In stability problems equations (5)-(8), with $Q^{\prime}=0$, and the associated boundary conditions are homogeneous and have solutions $\mathbf{B}^{\prime}, \mathbf{v}^{\prime}, \Theta^{\prime} \propto e^{\gamma t}$. The problem is then a generalised eigen- or critical-value problem ( $(4.1)$. Primes on perturbation fields are suppressed below.

The linearised equations are discretised in angle using vector spherical harmonic expansions (§2.2) of all vector fields and spherical harmonic expansions (\$2.1) of all scalar fields, for both the basic and perturbation states. Toroidal-poloidal representations ( $\$ 2.1)$ are also used, but only for the perturbation solenoidal vector fields, not the basic state fields. Thus there are five independent perturbation scalar fields: the temperature, and the toroidal and poloidal potentials of the magnetic field and the velocity. The resulting hybrid angular spectral equations (\$2.3) are more compact and less error prone to code, than if the toroidal-poloidal spectral interactions of products are fully expanded. Moreover, although there are ten products, only three subroutines are required to evaluate their angular dependence (§3). The subroutines calculate the three angular coupling integrals, which occur, in terms of $3 j-, 6 j$ - and $9 j$-symbols. Further, only six distinct combinations of perturbation radial functions and their derivatives occur, which greatly simplifies radial discretisation ( $\S 3)$. The code uses second-order finite-differences on a uniform radial grid. The resulting large-scale complex nonhermitian generalised eigen- and critical-value problems are solved using inverse and Newton-Raphson iteration methods, respectively. The code is tested against several models: rotating thermal convection (§4.1), kinematic roll-dynamos (§4.3) and magneto-convection $(\S 4.4)$. Concluding remarks are given in $\S 5$. 


\section{Hybrid vector spherical harmonic method}

\subsection{Toroidal-poloidal fields and spherical harmonics}

The solenoidal conditions (8) are satisfied by the toroidal-poloidal representations

$$
\mathbf{B}=\mathbf{T}\{T\}+\mathbf{S}\{S\}, \quad \mathbf{v}=\mathbf{T}\{t\}+\mathbf{S}\{s\},
$$

where $\mathbf{T}\{T\}:=\nabla \times\{T \mathbf{r}\}$ and $\mathbf{S}\{S\}:=\nabla \times \mathbf{T}\{S\}$. From the identity $\nabla \times \mathbf{S}\{S\}=\mathbf{T}\left\{-\nabla^{2} S\right\}$, it follows for the current and vorticity that

$$
\mu_{0} \mathbf{J}=\mathbf{T}\left\{-\nabla^{2} S\right\}+\mathbf{S}\{T\}, \quad \boldsymbol{\omega}=\mathbf{T}\left\{-\nabla^{2} s\right\}+\mathbf{S}\{t\} .
$$

The perturbation scalar fields are expanded in terms of (scalar) spherical harmonics. The spherical harmonic of degree $n$ and order $m$ in colatitude $\theta$ and east-longitude $\phi$ is defined for $m \geq 0$ by

$$
Y_{n}^{m}(\theta, \phi):=(-)^{m} \sqrt{\frac{(2 n+1)(n-m) !}{(n+m) !}} P_{n, m}(\cos \theta) e^{i m \phi},
$$

where

$$
P_{n, m}(z)=(-)^{n} \frac{\left(1-z^{2}\right)^{m / 2}}{2^{n} n !} \frac{d^{m+n}\left(1-z^{2}\right)^{n}}{d z^{m+n}}
$$

is the associated Legendre function of degree $n$ and order $m$. For $m<0$ the spherical harmonic of degree $n$ and order $m$ is defined by $Y_{n}^{m}:=(-)^{m}\left(Y_{n}^{-m}\right)^{*}$, where the star denotes complex conjugation. The spherical harmonics are orthonormal with respect to the innerproduct, $(f, g):=\frac{1}{4 \pi} \oint f g^{*} d \Omega$. A lowercase Greek subscript denotes 
the 2-index of a spherical harmonic. For example, $Y_{\alpha}$ denotes $Y_{n_{\alpha}}^{m_{\alpha}}$ and the orthonormalisation condition is $\left(Y_{\alpha}, Y_{\beta}\right)=\delta_{\alpha}^{\beta}$. Thus the poloidal and toroidal potentials, pressure and temperature have the spherical harmonic expansions

$$
f=\sum_{\alpha} f_{\alpha} Y_{\alpha}, \quad \text { for } f \in\{s, t, p, S, T, \Theta\} .
$$

Summations are over $n_{\alpha} \in \mathbb{N}$, and $m_{\alpha}=-n_{\alpha}, \ldots, n_{\alpha}$.

\subsection{Vector spherical harmonics}

The vector spherical harmonics used herein are defined by James [5]

$$
\mathbf{Y}_{n, n_{1}}^{m}:=(-)^{n-m} \sqrt{2 n+1} \sum_{m_{1}, \mu}\left(\begin{array}{ccc}
n & n_{1} & 1 \\
m & -m_{1} & -\mu
\end{array}\right) Y_{n_{1}}^{m_{1}} \mathbf{e}_{\mu}
$$

where $\mathbf{e}_{0}:=\mathbf{1}_{z}$ and $\mathbf{e}_{ \pm 1}:=\mp\left(\mathbf{1}_{x} \pm i \mathbf{1}_{y}\right) / \sqrt{2}$. In particular, $\mathbf{Y}_{n, 0}^{\mu}=$ $\delta_{n}^{1} \mathbf{e}_{\mu}$ and $\mathbf{Y}_{0,1}^{0}=-\mathbf{1}_{r}$. A lowercase Greek subscript on a vector field or its coefficient or argument denotes the 3-index of a vector spherical harmonic. Thus $\mathbf{Y}_{\gamma}$ denotes $\mathbf{Y}_{n_{\gamma}, n_{\gamma 1}}^{m_{\gamma}}$, otherwise $\gamma\left(\gamma_{1}\right)$ denotes $n_{\gamma}$ $\left(n_{\gamma 1}\right)$. If a 3 -index occurs on a scalar field or its coefficient the $n_{1}$ index is dropped. Vector spherical harmonics are orthonormal with respect to the inner-product $(\mathbf{F}, \mathbf{G}):=\frac{1}{4 \pi} \oint \mathbf{F} \cdot \mathbf{G}^{*} d \Omega$ of complex vector functions $\mathbf{F}$ and $\mathbf{G}$ of $\theta$ and $\phi$. Hence $\left(\mathbf{Y}_{\alpha}, \mathbf{Y}_{\beta}\right)=\delta_{\alpha, \beta}$. The basic-state and perturbation velocity, vorticity, magnetic induction and electric current fields are expanded in vector spherical harmonics,

$$
\mathbf{F}_{0}=\sum_{\alpha} F_{\alpha}^{0} \mathbf{Y}_{\alpha}, \quad \mathbf{F}=\sum_{\alpha} F_{\alpha} \mathbf{Y}_{\alpha}, \quad \mathbf{F}=\mathbf{v}, \boldsymbol{\omega}, \mathbf{B}, \mathbf{J}
$$

The summations are over $n_{\alpha} \in \mathbb{N}, n_{1 \alpha}=n_{\alpha}, n_{\alpha} \pm 1$ and $m_{\alpha}=$ $-n_{\alpha}, \ldots, n_{\alpha}$. 
The $Y$-expansions of $t$ and $s$ are related to the $\mathbf{Y}$-expansion of $\mathbf{v}$ by $v_{\gamma}=f_{v}(\gamma) \partial_{\gamma} s_{\gamma}$, if $\gamma_{1}=\gamma \pm 1$, and $v_{\gamma}=f_{v}(\gamma) t_{\gamma}$, if $\gamma_{1}=\gamma$, where

$$
f_{v}(\gamma):= \begin{cases}(\gamma+1) \sqrt{\gamma /(2 \gamma+1)}, & \text { if } \gamma_{1}=\gamma-1 \\ -i \sqrt{\gamma(\gamma+1)}, & \text { if } \gamma_{1}=\gamma \\ \gamma \sqrt{(\gamma+1) /(2 \gamma+1)}, & \text { if } \gamma_{1}=\gamma+1\end{cases}
$$

and $\partial_{n}^{n_{1}}:=\partial_{r}+(n+1) / r$, if $n_{1}=n-1$ and $\partial_{n}^{n_{1}}:=\partial_{r}-n / r$, if $n_{1}=$ $n+1$. In 3-index notation, $\partial_{\gamma}:=\partial_{n_{\gamma}}^{n_{1 \gamma}}$ and $\partial^{\gamma}:=\partial_{n_{1 \gamma}}^{n_{\gamma}}$. Similarly, the $Y$-expansions of $T$ and $S$ are related to the $\mathbf{Y}$-expansion of $\mathbf{B}$ by $B_{\gamma}=f_{B}(\gamma) \partial_{\gamma} S_{\gamma}$, if $\gamma_{1}=\gamma \pm 1$, and $B_{\gamma}=f_{B}(\gamma) T_{\gamma}$, if $\gamma_{1}=\gamma$, where $f_{B}(\gamma)=f_{v}(\gamma)$ for all $\gamma$. The $\mathbf{Y}$-components of the electric current are given by $\mu_{0} J_{\gamma}=f_{J}(\gamma) \partial_{\gamma} T_{\gamma}$, if $\gamma_{1}=\gamma \pm 1$, and $\mu_{0} J_{\gamma}=$ $f_{J}(\gamma) D_{\gamma} S_{\gamma}$, if $\gamma_{1}=\gamma$, where

$$
f_{J}(\gamma):= \begin{cases}(\gamma+1) \sqrt{\gamma /(2 \gamma+1)}, & \text { if } \gamma_{1}=\gamma-1 \\ i \sqrt{\gamma(\gamma+1)}, & \text { if } \gamma_{1}=\gamma \\ \gamma \sqrt{(\gamma+1) /(2 \gamma+1)}, & \text { if } \gamma_{1}=\gamma+1\end{cases}
$$

The $\mathbf{Y}$-components of the vorticity are related to the toroidal-poloidal velocity potentials by similar equations. The $\mathbf{Y}$-expansions of the temperature gradient $\mathbf{q}$ are related to the $Y$-expansion of $\Theta$ by $q_{\gamma}=f_{q}(\gamma) \partial_{\gamma} \Theta_{\gamma}$, if $\gamma_{1}=\gamma \pm 1$, and $q_{\gamma}=0$, if $\gamma_{1}=\gamma$, where $f_{q}$ is the temperature gradient field factor,

$$
f_{q}(\gamma):= \begin{cases}\sqrt{\frac{\gamma}{2 \gamma+1},}, & \text { if } \gamma_{1}=\gamma-1 \\ 0, & \text { if } \gamma_{1}=\gamma \\ -\sqrt{\frac{\gamma+1}{2 \gamma+1}}, & \text { if } \gamma_{1}=\gamma+1\end{cases}
$$




\subsection{The hybrid linearised spectral equations}

The hybrid linearised spectral momentum equations [3] for $t_{\gamma}$ and $s_{\gamma}$ are

$$
\begin{aligned}
\rho\left(\partial_{t}-\nu D_{\gamma}\right) t_{\gamma}= & \sum_{\substack{\alpha, \beta \\
\left(n_{1 \gamma}=n_{\gamma}\right)}}\left(J_{\alpha}^{0} B_{\beta} v_{\gamma}\right)-\left(B_{\beta}^{0} J_{\alpha} v_{\gamma}\right) \\
\rho\left(\partial_{t}-\nu D_{\gamma}\right) D_{\gamma} s_{\gamma}= & \sum_{\substack{\alpha, \beta \\
n_{1 \gamma}\left(\neq n_{\gamma}\right)}}\left(J_{\alpha}^{0} B_{\beta} v_{\gamma}\right)-\left(B_{\beta}^{0} \Theta_{\alpha} v_{\gamma}\right)-\rho 2\left(\Omega_{\alpha} v_{\beta} v_{\gamma}\right), \\
& -\rho \alpha_{\Theta}\left(g_{\alpha} \Theta_{\beta} v_{\gamma}\right)-\rho 2\left(\Omega_{\alpha} v_{\beta} v_{\gamma}\right),
\end{aligned}
$$

where $r^{2} D_{\gamma}:=r^{2} \partial_{r r}+2 r \partial_{r}-n_{\gamma}\left(n_{\gamma}+1\right)$.

The magnetic vector potential equation, $\partial_{t} \mathbf{A}=\eta \nabla^{2} \mathbf{A}+\mathbf{v} \times \mathbf{B}+$ $\nabla V$, where $\mathbf{B}=\nabla \times \mathbf{A}$, is used instead of the magnetic induction equation, since $\mathbf{F} \times \mathbf{G}$ is easier to handle than $\nabla \times(\mathbf{F} \times \mathbf{G})$. The magnetic vector potential spectral equations [3] for $S_{\gamma}$ and $T_{\gamma}$ are

$$
\begin{aligned}
\left(\partial_{t}-\eta D_{\gamma}\right) S_{\gamma} & =\sum_{\substack{\alpha, \beta \\
\left(n_{1 \gamma}=n_{\gamma}\right)}}\left(v_{\alpha}^{0} B_{\beta} A_{\gamma}\right)-\left(B_{\alpha}^{0} v_{\beta} A_{\gamma}\right), \\
\left(\partial_{\tau}-\eta D_{\gamma}\right) T_{\gamma} & =\sum_{\substack{\alpha, \beta \\
n_{1 \gamma}\left(\neq n_{\gamma}\right)}}\left(v_{\alpha}^{0} B_{\beta} A_{\gamma}\right)-\left(B_{\alpha}^{0} v_{\beta} A_{\gamma}\right) .
\end{aligned}
$$

The hybrid spectral linearised heat equation [3] for $\Theta_{\gamma}$ is

$$
\left(\partial_{t}-\kappa D_{\gamma}\right) \Theta_{\gamma}=\sum_{\alpha, \beta}-\left(v_{\alpha}^{0} q_{\beta} \Theta_{\gamma}\right)-\left(q_{\alpha}^{0} v_{\beta} \Theta_{\gamma}\right)
$$


The interaction terms are

$$
\begin{aligned}
& \left(J_{\alpha}^{0} B_{\beta} v_{\gamma}\right)=e_{v}(\gamma) f_{B}(\beta)\left(\mathbf{Y}_{\alpha} \times \mathbf{Y}_{\beta}, \mathbf{Y}_{\gamma}\right) \times \\
& \times \begin{cases}\partial^{\gamma}\left(J_{\alpha}^{0} \partial_{\beta} S_{\beta}\right), & \gamma_{1}=\gamma \pm 1, \beta_{1}=\beta \pm 1 ; \\
\partial^{\gamma}\left(J_{\alpha}^{0} T_{\beta}\right), & \gamma_{1}=\gamma \pm 1, \beta_{1}=\beta ; \\
J_{\alpha}^{0} \partial_{\beta} S_{\beta}, & \gamma_{1}=\gamma, \beta_{1}=\beta \pm 1 ; \\
J_{\alpha}^{0} T_{\beta}, & \gamma_{1}=\gamma, \beta_{1}=\beta ;\end{cases} \\
& \left(B_{\alpha}^{0} J_{\beta} v_{\gamma}\right)=e_{v}(\gamma) f_{J}(\beta)\left(\mathbf{Y}_{\alpha} \times \mathbf{Y}_{\beta}, \mathbf{Y}_{\gamma}\right) \times \\
& \times \begin{cases}\partial^{\gamma}\left(B_{\alpha}^{0} \partial_{\beta} T_{\beta}\right), & \gamma_{1}=\gamma \pm 1, \beta_{1}=\beta \pm 1 ; \\
\partial^{\gamma}\left(B_{\alpha}^{0} D_{\beta} S_{\beta}\right), & \gamma_{1}=\gamma \pm 1, \beta_{1}=\beta ; \\
B_{\alpha}^{0} \partial_{\beta} T_{\beta}, & \gamma_{1}=\gamma, \beta_{1}=\beta \pm 1 ; \\
B_{\alpha}^{0} D_{\beta} S_{\beta}, & \gamma_{1}=\gamma, \beta_{1}=\beta ;\end{cases} \\
& \left(g_{\alpha} \Theta_{\beta} v_{\gamma}\right)=\rho \alpha_{\Theta} e_{v}(\gamma)\left(\mathbf{Y}_{\alpha} Y_{\beta}, \mathbf{Y}_{\gamma}\right) \begin{cases}\partial^{\gamma}\left(g_{\alpha} \Theta_{\beta}\right), & \gamma_{1}=\gamma \pm 1 ; \\
g_{\alpha} \Theta_{\beta}, & \gamma_{1}=\gamma ;\end{cases} \\
& \left(v_{\alpha}^{0} q_{\beta} \Theta_{\gamma}\right)=f_{q}(\beta)\left(\mathbf{Y}_{\alpha} \cdot \mathbf{Y}_{\beta}, Y_{\gamma}\right) \begin{cases}v_{\alpha}^{0} \partial_{\beta} \Theta_{\beta}, & \beta_{1}=\beta \pm 1 \\
0, & \beta_{1}=\beta .\end{cases}
\end{aligned}
$$

The terms $\left(\Omega_{\alpha}^{0} v_{\beta} v_{\gamma}\right),\left(v_{\alpha}^{0} B_{\beta} A_{\gamma}\right)$ and $\left(B_{\alpha}^{0} v_{\beta} A_{\gamma}\right)$ have the same form as $\left(J_{\alpha}^{0} B_{\beta} v_{\gamma}\right)$, and are obtained by making the obvious substitutions. The $\left(q_{\alpha}^{0} v_{\beta} \Theta_{\gamma}\right)$ has the same form as $\left(v_{\alpha}^{0} q_{\beta} \Theta_{\gamma}\right)$. Each term is a product of an equation factor, a perturbation field factor, a coupling integral and a radial expression. The velocity, magnetic and temperature equation factors are

$$
e_{v}(\gamma):= \begin{cases}1 / \sqrt{\gamma(2 \gamma+1)}, & \text { if } \gamma_{1}=\gamma-1 ; \\ i / \sqrt{\gamma(\gamma+1)}, & \text { if } \gamma_{1}=\gamma \\ 1 / \sqrt{(\gamma+1)(2 \gamma+1)}, & \text { if } \gamma_{1}=\gamma+1\end{cases}
$$

$e_{B}(\gamma)=(-)^{\gamma-\gamma_{1}} e_{v}(\gamma)$ and $e_{\Theta}=1$. 
The coupling integrals of these three harmonics, $\left(\mathbf{Y}_{\alpha} \times \mathbf{Y}_{\beta}, \mathbf{Y}_{\gamma}\right)$, $\left(\mathbf{Y}_{\alpha} Y_{\beta}, \mathbf{Y}_{\gamma}\right)$ and $\left(\mathbf{Y}_{\alpha} \cdot \mathbf{Y}_{\beta}, Y_{\gamma}\right)$, have been evaluated in closed form [5] in terms of $3 j-, 6 j$ - and $9 j$-symbols, which are readily evaluated. The series (10) and (11), and the equations (15)-(19), are truncated at $n=N_{n}$.

\section{Code structure and radial discretisation}

There are three general routines for cross-products $\mathbf{F}_{0} \times \mathbf{F}$, dot products $\mathbf{F}_{0} \cdot \mathbf{F}$ and scalar multiplications $f \mathbf{F}_{0}$. The routine for cross-products calculates the angular part, that is, the coupling integral $\left(\mathbf{Y}_{\alpha} \times \mathbf{Y}_{\beta}, \mathbf{Y}_{\gamma}\right)$, and calls two common routines to evaluate the radial part and the position of an entry in the relevant matrix. This one routine evaluates all seven cross products, $\mathbf{v}_{0} \times \mathbf{B}, \mathbf{v} \times \mathbf{B}_{0}$, $\mathbf{1}_{z} \times \mathbf{v}, \mathbf{J} \times \mathbf{B}_{0}, \mathbf{J}_{0} \times \mathbf{B}$. The dot product and scalar multiplication routines calculate the coupling integrals, $\left(\mathbf{Y}_{\alpha} \cdot \mathbf{Y}_{\beta}, Y_{\gamma}\right)$ and $\left(\mathbf{Y}_{\alpha} Y_{\beta}, \mathbf{Y}_{\gamma}\right)$, but call the same radial and position routines. They evaluate $\mathbf{v}_{0} \cdot \nabla \Theta, \mathbf{v} \cdot \nabla \Theta_{0}$ and $\mathbf{g} \Theta$. There is also one routine for the scalar and vector Laplacians, $\nabla^{2} \Theta, \nabla^{2} \mathbf{A}, \nabla^{2} \mathbf{v}$.

Two symmetries are exploited to reduce the numerical problem size. The first is the axisymmetry of the basic state, which decouples the spherical harmonic orders $m$ of the perturbation fields. Eigenand critical solutions are of the form, $f(r, \theta, \phi)=\widetilde{f}(r, \theta) e^{i m \phi}$, for $f \in$ $\{s, t, S, T, \Theta\}$. The order $m$ enters as a parameter, which is fixed apriori, eliminating one independent variable, $\phi$. Since the fields are real, only $m \geq 0$ is considered. The second is symmetry under reflection in the equator, that is, parity: even/odd $(e / o)$ for scalars and dipole/quadrupole $(d / q)$ for vectors. A scalar $f$ is even/odd, if $f(r, \pi-\theta, \phi)= \pm f(r, \theta, \phi)$; a vector field $\mathbf{F}$ is dipole/quadrupole, if 
$F_{r}(r, \pi-\theta, \phi)=\mp F_{r}(r, \theta, \phi), F_{\theta}(r, \pi-\theta, \phi)= \pm F_{\theta}(r, \theta, \phi), F_{\phi}(r, \pi-$ $\theta, \phi)=\mp F_{\phi}(r, \theta, \phi)$. Since $Y_{n}^{m}(\pi-\theta, \phi)=(-)^{n-m} Y_{n}^{m}(\theta, \phi)$, the field $\mathbf{B}(\mathbf{v})$ is dipole/quadrupole, if $S_{n}^{m}=0\left(s_{n}^{m}=0\right)$ for $n-m$ even/ odd and $T_{n}^{m}=0\left(t_{n}^{m}=0\right)$ for $n-m$ odd/even. $\Theta$ is even/odd, if $\Theta_{n}^{m}=0$ for $n-m$ odd/even. The problem decouples into two groups, $\left(\mathbf{B}_{q}, \mathbf{v}_{q}, \Theta_{e}\right)$ and $\left(\mathbf{B}_{d}, \mathbf{v}_{q}, \Theta_{e}\right)$, if $\mathbf{v}_{d}=\mathbf{0}, \Theta_{o}=0$ and $\mathbf{g}_{d}=\mathbf{0}$.

Only six types of radial expressions occur,

$$
f^{0} \partial_{\beta} f, f^{0} D_{\beta} f, \partial^{\gamma}\left(f^{0} f\right), \partial^{\gamma}\left(f^{0} \partial_{\beta} f\right), \partial^{\gamma}\left(f^{0} D_{\beta} f\right), D_{\gamma} D_{\gamma} f,
$$

apart from the trivial $f^{0} f$. The compact form of these expressions greatly simplifies radial discretisation. Second-order finitedifferences on a uniform grid, $r_{j}=j / N_{r}, j=1, \ldots, N_{r}$, are used. The centred schemes,

$$
\begin{aligned}
f_{0}^{(1)} & =\frac{-f_{-1}+f_{1}}{2 h}-\frac{1}{6} f^{(3)}(\eta) h^{2}, \\
f_{0}^{(2)} & =\frac{f_{-1}-2 f_{0}+f_{1}}{h^{2}}-\frac{1}{12} f^{(4)}(\eta) h^{2}, \\
f_{0}^{(3)} & =\frac{-f_{-2}+2 f_{-1}-2 f_{1}+f_{2}}{2 h^{3}}-\frac{1}{4} f^{(5)}(\eta) h^{2}, \\
f_{0}^{(4)} & =\frac{f_{-2}-4 f_{-1}+6 f_{0}-4 f_{1}+f_{2}}{h^{4}}-\frac{1}{6} f^{(6)}(\eta) h^{2},
\end{aligned}
$$

where the subscripts indicate relative position, are used at the interior points $r_{j}$, where $j=1, \ldots, N_{r}-1$ for (20), (21) and $j=$ $1, \ldots, N_{r}-2$ for (22), (23). The schemes must be adapted near $r=0$. If a function $f$ is analytic at the origin, then $f_{n}^{m}$ is of the form $f_{n}^{m}=r^{n} \sum_{k=0}^{\infty} a_{k} r^{2 k}$ near $r=0$, where the $a_{k}$ are independent of $r$, and hence $f_{n}^{m}(-r)=(-)^{n} f_{n}^{m}(r)$. This identity is used to fold the schemes from negative grid points back to positive points. Note that the poloidal-toroidal potentials of an analytic vector field are analytic. 
The centred schemes are supplemented by one-sided right boundary schemes near $r=1$,

$$
\begin{aligned}
f_{0}^{(1)} & =\frac{f_{-2}-4 f_{-1}+3 f_{0}}{2 h}+\frac{1}{3} f^{(3)}(\eta) h^{2}, \\
f_{0}^{(2)} & =\frac{-f_{-3}+4 f_{-2}-5 f_{-1}+2 f_{0}}{h^{2}}+\frac{11}{12} f^{(4)}(\eta) h^{2}, \\
f_{0}^{(3)} & =\frac{3 f_{-4}-14 f_{-3}+24 f_{-2}-18 f_{-1}+5 f_{0}}{2 h^{3}}+\frac{7}{4} f^{(5)}(\eta) h^{2}, \\
f_{0}^{(3)} & =\frac{f_{-3}-6 f_{-2}+12 f_{-1}-10 f_{0}+3 f_{1}}{2 h^{3}}+\frac{1}{4} f^{(5)}(\eta) h^{2},
\end{aligned}
$$

if the boundary conditions fix $f$, and

$$
\begin{aligned}
f_{0}^{(2)}= & \frac{-f_{-2}+8 f_{-1}-7 f_{0}+6 h f_{0}^{(1)}}{2 h^{2}}+\frac{1}{6} f^{(4)}(\eta) h^{2}, \\
f_{0}^{(3)}= & \frac{f_{-3}-6 f_{-2}+15 f_{-1}-10 f_{0}+6 h f_{0}^{(1)}}{h^{3}}+\frac{11}{20} f^{(5)}(\eta) h^{2}, \\
f_{0}^{(3)}= & \frac{-f_{-2}+9 f_{0}-8 f_{1}+6 h f_{1}^{(1)}}{3 h^{3}}-\frac{3}{20} f^{(5)}(\eta) h^{2}, \\
f_{0}^{(4)}= & \frac{-3 f_{-3}+32 f_{-2}-108 f_{-1}+192 f_{0}-113 f_{1}+60 h f_{1}^{(1)}}{12 h^{4}} \\
& -\frac{1}{21} f^{(7)}(\eta) h^{3},
\end{aligned}
$$

if the boundary conditions fix $f^{(1)}$. Schemes (24)-(26), (28) and (29) are applied at $r_{N_{r}}$; schemes (27), (30) and (31) are applied at $r_{N_{r}-1}$. The code produces three large, sparse, banded, complex matrices $\mathbf{A}$, $\mathbf{B}$ and $\mathbf{C}$. The bandwidth of the matrices for the most general problem is minimised, if the spherical harmonic coefficients, $S_{n}^{m}, T_{n}^{m}, s_{n}^{m}$, $t_{n}^{m}, \Theta_{n}^{m}$, at each gridpoint are blocked together. If the problem uses the difference schemes (22) and (23), then the matrices A, B and $\mathbf{C}$ are block pentadiagonal, apart from a possible third subdiagonal boundary block, if schemes (25), (27), (29) and (31) are used, and 
fourth subdiagonal boundary block, if (26) is used. The matrices are $N \times N$, where

$$
N=\sum_{f \in\{S, T, s, t, \Theta\}}\left(N_{r}-b_{f}\right)\left(N_{n}-m+1\right) p_{f},
$$

with $b_{f}=1$, if $f$ is fixed at $r=1$ by the boundary condition and $b_{f}=0$ otherwise, and with $p_{f}=0.5$ if $f$ is even or odd and $p_{f}=1$ otherwise.

\section{Models and results}

\subsection{Methods for eigen- and critical-value problems}

The code produces the matrices $\mathbf{A}, \mathbf{B}$ and $\mathbf{C}$ for two kinds of problem. The first is the generalised eigenvalue problem,

$$
(\mathbf{A}+R \mathbf{C}) \mathbf{x}=\gamma \mathbf{B x},
$$

for the growth rate $\gamma$ of largest real part and the associated eigenvector $\mathbf{x}$, where $R$ is a dimensionless parameter. The second is the critical value problem,

$$
(\mathbf{A}+R \mathbf{C}) \mathbf{x}=i \omega \mathbf{B} \mathbf{x},
$$

for the critical value of the real parameter $R$ and the critical frequency $\omega=\Im \gamma$, that is, the values where the real part of the growth rate $\gamma$ vanishes, $\Re \gamma=0$. Problem (32) is solved using generalised inverse iteration,

$$
\mathbf{y}_{n}=\left(\mathbf{A}+R \mathbf{C}-\gamma_{0} \mathbf{B}\right)^{-1} \mathbf{B} \mathbf{x}_{n-1}, \quad \gamma_{n}=\gamma_{0}+\hat{y}_{n}, \quad \mathbf{x}_{n}=\hat{y}_{n}^{-1} \mathbf{y}_{n},
$$


where $\hat{y}_{n}$, which may be complex, is the element of $\mathbf{y}_{n}$ of largest magnitude, and $\left(\gamma_{0}, \mathbf{x}_{0}\right)$ is an estimate of $(\gamma, \mathbf{x})$. Problem (33), which is non-linear in the unknowns, $(R, \omega, \mathbf{x})$, as well as underdetermined since it is homogeneous in $\mathbf{x}$, is supplemented by an linear normalising equation in $\mathbf{x}$ and solved by Newton-Raphson iteration [7].

\subsection{Rotating thermal convection}

The basic state for rotating thermal convection is $\mathbf{B}_{0}=\mathbf{0}, \mathbf{v}_{0}=\mathbf{0}$ and the steady conduction temperature $\Theta_{0}$. In the present model, the volume heat production is constant, $\Theta_{0}$ vanishes on the boundary $r=a$, and gravity $\mathbf{g}=-g_{a} \mathbf{r} / a$. In terms of the boundary temperature gradient $-\beta$, the heat production is $Q_{0}=3 \kappa \rho c_{p} \beta / a$ and the temperature is $\Theta_{0}=\beta\left(a^{2}-r^{2}\right) / 2 a$. The perturbation magnetic field $\mathbf{B}$ simply decays, since $\mathbf{v}_{0}=\mathbf{0}$, and so is omitted. Further, there is no surplus heat production, $Q=Q_{0}$.

The remaining problem is non-dimensionalised using the radial length scale $a$, the viscous diffusion time scale $a^{2} / \nu$, the viscous velocity scale $\nu / a$ and the temperature scale $a \beta$. The dimensionless equations for the perturbation velocity and temperature, $\mathbf{v}$ and $\Theta$ are

$$
\begin{aligned}
E \partial_{t} \mathbf{v}+\mathbf{1}_{z} \times \mathbf{v} & =-\nabla P+R \Theta \mathbf{r}+E \nabla^{2} \mathbf{v} \\
\partial_{t} \Theta-\mathbf{v} \cdot \mathbf{r} & =\operatorname{Pr}^{-1} \nabla^{2} \Theta
\end{aligned}
$$

where $E:=\nu / 2 \Omega a^{2}, R:=\alpha g_{a} a^{2} \beta / 2 \Omega \nu$ and $\operatorname{Pr}:=\nu / \kappa$ are the Ekman, a modified Rayleigh and Prandtl numbers. On the boundary the velocity satisfies the no-slip condition, $\mathbf{v}=\mathbf{0}$, and the temperature vanishes, $\Theta=0$. Thus

$$
s_{n}^{m}=\partial_{r} s_{n}^{m}=t_{n}^{m}=\Theta_{n}^{m}=0, \quad \text { at } r=1 .
$$


TABLE 1: Results for rotating thermal convection model.

\begin{tabular}{|rrrr|rcc|}
\hline$E$ & $m$ & $N_{n}$ & $N_{r}$ & $\Im \gamma$ & $R$ & $R[6]$ \\
\hline $10^{-2}$ & 1 & 10 & 100 & 0.10434 & 180.74 & 180.74 \\
$10^{-2}$ & 2 & 10 & 100 & 0.097868 & 157.83 & 157.88 \\
$10^{-2}$ & 3 & 10 & 100 & 0.089180 & 186.20 & 186.25 \\
$5.7735 \times 10^{-3}$ & 1 & 10 & 200 & 0.065721 & 159.00 & 159.10 \\
$5.7735 \times 10^{-3}$ & 2 & 10 & 200 & 0.066732 & 129.02 & 129.06 \\
$5.7735 \times 10^{-3}$ & 3 & 10 & 200 & 0.070462 & 137.26 & 137.30 \\
$3.1623 \times 10^{-3}$ & 1 & 10 & 200 & 0.025556 & 143.94 & 143.98 \\
$3.1623 \times 10^{-3}$ & 2 & 10 & 200 & 0.029171 & 111.04 & 111.11 \\
$3.1623 \times 10^{-3}$ & 3 & 10 & 200 & 0.040854 & 111.96 & 112.02 \\
\hline
\end{tabular}

Critical values of the modified Rayleigh number for the test model [6] with $\operatorname{Pr}=1$ are shown in Table 1 for the quadrupole modes. The associated critical frequencies are also given. In [6], which treats only rotating thermal convection in a sphere, the toroidal-poloidal momentum equations and the heat equation are discretised using a Galerkin method in radius and angle. The radial basis functions are linear combinations of modified spherical Bessel functions and the angle basis functions are spherical harmonics. For a given radial truncation level the method of [6] is more accurate than the radial finite-difference/angle Galerkin method used herein, but it is also substantially more difficult to apply and is not as general.

\subsection{Kinematic dynamos with axisymmetric flows}

The basic state for kinematic dynamos is $\mathbf{B}_{0}=\mathbf{0}, \Theta_{0}=0$ with a prescribed flow $\mathbf{v}_{0}$, which is axisymmetric. The magnetic field $\mathbf{B}$ is due to the electric currents generated by the motion $\mathbf{v}_{0}$ of a spher- 
ical conducting fluid $V$ of radius $a$ and uniform magnetic diffusivity $\eta$. The exterior is electrically insulating and the dynamo is selfexciting, that is, there are no sources of magnetic field at infinity. The perturbation velocity, temperature, Navier-Stokes equation and heat equation are omitted. The equations are non-dimensionalised using the radial length scale $a$, a typical flow speed $v_{0}$ and the magnetic diffusion time scale, $a^{2} / \eta$. The magnetic field $\mathbf{B}$ is governed by

$$
\begin{aligned}
& \partial_{t} \mathbf{B}=\nabla^{2} \mathbf{B}+R \nabla \times\left(\mathbf{v}_{0} \times \mathbf{B}\right), \quad \text { in } V ; \\
& \nabla \cdot \mathbf{B}=0, \quad \text { in } E^{3} ; \\
& \nabla \times \mathbf{B}=\mathbf{0}, \quad \text { in } \widehat{V} ; \\
& {[\mathbf{B}]_{\partial V}=\mathbf{0}, \quad \text { on } \partial V ; \quad \mathbf{B} \rightarrow \mathbf{0}, \quad \text { as } r \rightarrow \infty ; }
\end{aligned}
$$

where $R:=a v_{0} / \eta$ is the magnetic Reynolds number.

The equations in the insulating exterior $\widehat{V}$ reduce to $T=\nabla^{2} S=$ 0 and the continuity condition on $\partial V$ becomes $[T]=0=[S]=\left[\partial_{r} S\right]$ at $r=1$. Substitution of the expansions $(10)$ gives $T_{n}^{m}(r, t)=$ 0 and $S_{n}^{m}(r, t)=S_{n}^{m}(1, t) r^{-n-1}$ in $r>1$. Eliminating $S_{n}^{m}(1, t)$, using the continuity conditions, yields the local poloidal and toroidal boundary conditions,

$$
\partial_{r} S_{n}^{m}+(n+1) S_{n}^{m}=T_{n}^{m}=0, \quad \text { at } r=1 .
$$

The magnetic (Ohmic) free-decay case $R_{m}=0$ has analytic solution

$$
T_{n k}^{m}=j_{n}\left(j_{n, k} r\right) e^{-j_{n, k}^{2} t}, \quad S_{n k}^{m}=j_{n}\left(j_{n-1, k} r\right) e^{-j_{n-1, k}^{2} t},
$$

for $r<1, n, k \in \mathbb{N}^{+}$, where $j_{n}$ is the $n$th order spherical Bessel function of the first kind and $j_{n, k}$ is the $k$ th zero of $j_{n}$. The slowest decaying poloidal modes are the $n=1, k=1(m=-1,0,1)$ modes with (dimensional) decay time $t_{P}=a^{2} / j_{01}^{2} \eta, j_{01}=\pi$. The slowest 
TABle 2: Dynamo growth rates for flow $s_{2} t_{1}$, with $m=1, N_{n}=12$ and $N_{r}=100$.

\begin{tabular}{|rr|rrrr|}
\hline \multicolumn{1}{|c|}{$\epsilon$} & \multicolumn{1}{c|}{$R$} & \multicolumn{1}{c}{$\Re \gamma$} & \multicolumn{1}{c}{$\Im \gamma$} & $\Re \gamma[1]$ & $\Im \gamma[1]$ \\
\hline 0.1 & 120 & 0.9955 & -31.325 & 0.996 & -31.31 \\
0.13 & 94.9385 & 0 & -18.2153 & & \\
0.13 & 100 & 0.5552 & -18.7935 & & \\
\hline
\end{tabular}

decaying toroidal modes are the $n=1, k=1(m=-1,0,1)$ modes with decay time $t_{T}=a^{2} / j_{11}^{2} \eta, j_{11}=4.493409$. (For the Earth $t_{P}=19,500 \mathrm{yr}$ and $t_{T}=9,500 \mathrm{yr}$ excluding a primordial magnetic field.)

The kinematic dynamo test model is a double-roll $s_{2} t_{1}$ flow [1],

$$
\mathbf{v}_{0}=\mathbf{t}_{1}^{0}+\epsilon \mathbf{s}_{2}^{0}, \quad t_{1}^{0}=\sin (\pi r) / \sqrt{3}, \quad s_{2}^{0}=r \sin (\pi r) / \sqrt{5},
$$

where $\epsilon$ is a disposable parameter. The non-zero basic state vector spherical harmonic coefficients are

$$
\begin{aligned}
& v_{1,1}^{0}=-i \sqrt{2 / 3} \sin \pi r, \\
& v_{2,1}^{0}=\epsilon 3 \sqrt{2} / 5(\pi r \cos \pi r+4 \sin \pi r), \\
& v_{2,3}^{0}=\epsilon 2 \sqrt{3} / 5(\pi r \cos \pi r-\sin \pi r) .
\end{aligned}
$$

Selected results are shown in Table 2 for $m=1$ quadrupole solutions. In [1] the magnetic induction equation is discretised using toroidal and poloidal fields, a spherical harmonic Galerkin method in angle and second-order finite-differences in radius with Richardson extrapolation, but a lower order radial boundary scheme. The critical magnetic Reynolds number 94.9385 for $\epsilon=0.13$ compares with the value 95 given in [1]. 


\subsection{Rotating magnetoconvection}

The basic state for magnetoconvection is $\mathbf{v}_{0}=\mathbf{0}$ with the steady conduction temperature $\Theta_{0}$ and a prescribed magnetic field $\mathbf{B}_{0}$, which is axisymmetric. The thermal convection of a rotating spherical conducting fluid $V$ of radius $a$, which is driven by the temperature gradient, is modified by the imposed magnetic field $\mathbf{B}_{0}$. The perturbation magnetic field is due to the electric currents in $V$. The diffusivities, $\nu, \kappa$ and $\eta$, are constant. The exterior is electrically insulating and the magnetic field perturbations are self-exciting. In the present model, the volume heat production is constant, $\Theta_{0}$ vanishes on the boundary $r=a$ and gravity $\mathbf{g}=-g_{a} \mathbf{r} / a$. The temperature is $\Theta_{0}=\beta\left(a^{2}-r^{2}\right) / 2 a$. There is no surplus heat production, $Q=0$.

The variables are non-dimensionalised using the radial length scale $a$, the magnetic diffusion time scale $a^{2} / \eta$, the magnetic velocity scale $\eta / a$, the temperature scale $a \beta$ and the magnetic scale $B_{0}$, where $B_{0}$ is a typical value of $\mathbf{B}_{0}$. The dimensionless equations for the perturbation velocity, magnetic field and temperature, $\mathbf{v}, \mathbf{B}, \Theta$, are

$$
\begin{aligned}
E_{\eta} \partial_{t} \mathbf{v}+\mathbf{1}_{z} \times \mathbf{v} & =-\nabla P+\Lambda \mathbf{J}_{0} \times \mathbf{B}+R \Theta \mathbf{r}+E \nabla^{2} \mathbf{v}, \\
\partial_{t} \mathbf{B} & =\nabla^{2} \mathbf{B}+\nabla \times\left(\mathbf{v} \times \mathbf{B}_{0}\right) \\
\partial_{t} \Theta-\mathbf{v} \cdot \mathbf{r} & =q \nabla^{2} \Theta
\end{aligned}
$$

where $E_{\eta}:=\eta / 2 \Omega a^{2}, \Lambda:=B_{0}^{2} / 2 \Omega \eta \mu_{0} \rho, R:=\alpha g_{a} a^{2} \beta / 2 \Omega \eta, E:=$ $\nu / 2 \Omega a^{2}$ and $q:=\kappa / \eta$ are the magnetic Ekman, Elsasser, a modified Rayleigh and Roberts numbers. On the boundary the velocity satisfies the no-slip condition, $\mathbf{v}=\mathbf{0}$, the temperature vanishes, $\Theta=0$, and the magnetic field matches to a potential field vanishing at infinity, equations (34)-(36). Thus

$$
\partial_{r} S_{n}^{m}+(n+1) S_{n}^{m}=T_{n}^{m}=s_{n}^{m}=\partial_{r} s_{n}^{m}=t_{n}^{m}=\Theta_{n}^{m}=0 \text {, at } r=1 .
$$


TABLE 3: Critical Rayleigh number for the magneto-convection model.

\begin{tabular}{|rr|rr|}
\hline$N_{n}$ & $N_{r}$ & \multicolumn{1}{c|}{$R$} & $\Im \gamma$ \\
\hline 10 & 50 & 110.03291 & -43.14997 \\
10 & 20 & 113.3 & $-42.35[2]$ \\
20 & 20 & 115.0 & $-43.25[4]$ \\
\hline
\end{tabular}

In the test model $[2,4]$ the basic state magnetic field is purely toroidal, $\mathbf{B}_{0}=\sin \theta \mathbf{1}_{\phi}$, and the non-zero basic state vector spherical harmonic coefficients are $B_{1,1}^{0}=-i \sqrt{2 / 3} r$ and $J_{1,1}^{0}=2$. The critical $(\Re \gamma=0)$ modified Rayleigh number $R$ is shown in Table 3 for $\Lambda=0.5$ and $m=2$, and compared with less general methods [2] and [4]. The former [2] used a Galerkin method in radius and angle and was restricted only to the basic state given above; the latter [4] used a less accurate method, namely finite-differences in both $r$ and $\theta$ with the $\phi$-dependence separated out.

\section{Concluding remarks}

A hybrid spectral code for the linearised magnetohydrodynamic equations in a sphere, which uses vector spherical harmonics and toroidal-poloidal fields, is described. The basic state is axisymmetric. Good agreement is obtained with published results for three model problems.

Acknowledgements: This research has been supported in part by an Australian Research Council Institutional Grant. 


\section{References}

[1] M. L. Dudley and R. W. James. Time-dependent kinematic dynamos with stationary flows. Proc. R. Soc. Lond. A 425:407-429, 1989. C439

[2] D. R. Fearn. Thermal and magnetic instabilities in a rapidly rotating fluid sphere. Geophys. Astrophys. Fluid Mech. 14:103-126, 1979. C441

[3] D. J. Ivers and C.G. Phillips. Scalar and vector spherical harmonic spectral equations of rotating non-linear and linearised magnetohydrodynamics, in preparation. 2001. C430

[4] D. R. Fearn and M. R. E. Proctor. Hydromagnetic waves in a differentially rotating sphere. J. Fluid Mech. 128:1-20, 1983. C441

[5] R. W. James. New tensor spherical harmonics for application to the partial differential equations of mathematical physics. Phil. Trans. R. Soc. Lond. A 281:195-221, 1976. C428, C432

[6] P. H. Roberts. On the thermal instability of a rotating-fluid sphere containing heat sources. Phil. Trans. R. Soc. Lond. A 263:93-117, 1968. C437

[7] K. Zhang. On coupling between the Poincaré equation and the heat equation. J. Fluid Mech. 268:211-229, 1994. C436 